\section{Efficacy of associative versus dissociative strategies in producing extreme affective ratings of a stimulus person*}

\author{
RICHARD R. IZZETT† and WALTER LEGINSKI \\ State University of New York, College at Oswego, Oswego, N.Y. 13126
}

In a test of a hypothesis that conveying information about a stimulus person via associative linkage would produce more extreme affective ratings of the stimulus person than conveying information via dissociative linkage, 124 Ss were assigned randomly to one of four treatment conditions: (1) positive information conveyed via an associative link; (2) positive information conveyed via a dissociative link; (3) negative information conveyed via an associative link; and (4) negative information conveyed via a dissociative link. In support of the hypothesis, results indicate that conveying information via associative linkage produces more extreme affective ratings of the stimulus person.

According to various balance theories (Abelson \& Rosenberg, 1958; Heider, 1958; Osgood \& Tannenbaum, 1955), if a source is associatively linked with a positive trait word (e.g., Mr. X is reasonable) or dissociatively linked with a negative trait word (e.g., $\mathrm{Mr}$. $\mathrm{X}$ is not unreasonable), theoretically there should be a positive change in the evaluation of the source. Alternatively, if a source is associatively linked with a negative trait word (e.g., Mr. X is unreasonable) or dissociatively linked with a positive trait word (e.g., Mr. X is not reasonable), there should be a negative change in the evaluation of the source.

This study focuses on the relative efficacy of an associative vs dissociative strategy in conveying information about a source. Specifically, the study is concerned with the extremeness of S's ratings of a source that has had positive or negative information conveyed about it via an associative (e.g., is) vs dissociative (e.g., is not) link.

Fishbein (1963) has demonstrated that extremity of affective responses toward an attitudinal object is, in part, a function of the amount of information conveyed about the attitudinal object. Because of the more definitive nature of an associative link (is) as compared to a dissociative link (is not), it is believed that more information will be conveyed about a source when that source has information conveyed about it via an associative link. Therefore, it is hypothesized that conveying information about a stimulus person

*This research was supported by a fellowship from the University Awards Committee of the Research Foundation of State University of New York to the senior author.

tRequest for reprints should be sent to Richard R. Izzett, Psychology Department, State University of New York, College at Oswego, Oswego, N.Y. 13126. via an associative link will be more effective than conveying information via a dissociative link in producing extreme affective responses.

\section{SUBJECTS AND DESIGN}

One hundred and twenty-four students enrolled in an introductory psychology course were randomly assigned to one of four treatment conditions generated by the two types of linkage-associative and dissociative-and two type of trait words-positive and negative. There were 31 Ss in each treatment condition, and all Ss were run in groups of 11 to 25 .

An associative link was operationally defined as the word is, while a dissociative link was operationally defined as the words is not.

Positive information was operationally defined as the total unit of an associative link associated with a positive trait word (e.g., Mr. X is reasonable) or as the total unit of a dissociative link associated with a negative trait word (e.g., Mr. X is not unreasonable). Negative information was operationally defined as the total unit of an associative link associated with a negative trait word (Mr. $\mathrm{X}$ is unreasonable) or as the total unit of a dissociative link associated with a positive trait word (e.g., Mr. X is not reasonable).

\section{STIMULUS MATERIAL}

Six trait words, three with positive ratings and three with negative ratings, were selected from Anderson's (1968a) list of personality trait words. The three positive trait words were thoughtful, trustful, and reasonable, while the three negative trait words were thoughtless, distrustful, and unreasonable. These particular trait words were selected in order to have stimulus information that deviated to the same degree from an affectively neutral point, be it in a positive or negative direction. For example, the trait word reasonable, according to Anderson's normative ratings, deviates 2 points in the positive direction from the neutral point (Scale Position 4) on a 7-point scale, while the trait word unreasonable deviates 2.03 points from the neutral point in the negative direction. Thus, when one compares the effectiveness of conveying information via an associative link (e.g., Mr. X is reasonable) to conveying information via a dissociative link (e.g., Mr. X is not unreasonable) in producing extreme affective ratings of the attitudinal object, any difference between the two strategies will be due to the types of linkage and not the extremity of the trait word.

\section{PROCEDURE}

After all Ss arrived in the experimental room, each $S$ was handed a four-page booklet. Randomization of Ss to treatment conditions was obtained by shuffling the four types of booklets representing each treatment condition prior to the Ss' entering the experimental room.

The first page of each booklet contained a series of instructions informing the $S$ that the remainder of the booklet contained a number of brief one-sentence descriptions of a few hypothetical men-one description per page. Ss were instructed to read the description of the hypothetical man and then rate him on a 7 -point bipolar scale set up in semantic differential form on the basis of how much they would like or dislike a person who possessed such a trait.

The Ss were also instructed to pay close attention to the endpoints of the evaluation scale and not to misread the endpoints, as some Ss accidentally do. To aid the $S s$ in making careful evaluations, all Ss were given two practice items. Upon completion of the practice items, the $E$ again asked the Ss to recheck their evaluations, making sure that they did not misread the endpoints of the scale. Following this, all Ss were instructed to complete the remainder of the booklet. At this point, Ss were asked to take their task seriously and to give honest and truth ful evaluations.

In all treatment conditions, stimulus information was presented in the format: Mr. X (Y or Z) linkage (is or is not) trait word (positive or negative). Each of the three experimental descriptive statements and the two practice descriptive statements in each booklet were all of the same format, representing one of the four treatment conditions. The order of each of the three experimental descriptive statements in each booklet was also randomly varied.

$$
\text { RESULTS }
$$

In a test of the hypothesis, 
Table 1

Mean Extremity Scores for Treatment Conditions

\begin{tabular}{lccc}
\hline & $\begin{array}{c}\text { Positive } \\
\text { Information }\end{array}$ & $\begin{array}{c}\text { Negative } \\
\text { Information }\end{array}$ & $\begin{array}{c}\text { Row } \\
\text { Mean }\end{array}$ \\
\hline Associative Link & 6.51 & 6.61 & 6.56 \\
Dissociative Link & 5.19 & 5.48 & 5.33 \\
Column Mean & 5.85 & 6.04 & \\
\hline
\end{tabular}

extremeness of S's ratings of the stimulus person was evaluated. For each of the three ratings that each $S$ gave, an extremity score was obtained by determining the distance between the S's ratings and the neutral point on the 7-point bipolar scale. For example, if a $S$ evaluated the stimulus object as a 7 on the 7-point bipolar scale of like-dislike, he was given an extremity score of 3 , since the neutral point had a rating of 4. Extremity scores for each of the S's three evaluations were then summed, and then the analysis of variance was performed on these summated extremity scores. In those conditions where positive information was conveyed about the stimulus person-an associative link with a positive trait word or a dissociative link with a negative trait word-the extremity score for Ss' ratings were positive if they were greater than 4 and negative if less than 4 . In those conditions where negative information was conveyed about the stimulus person-an associative link with a negative trait word or a dissociative link with a positive trait word-the extremity scores for Ss' ratings were positive if they were less than 4 and negative if greater than 4.

Mean extremity scores for treatment conditions are presented in Table 1. In support of the hypothesis, a main effect for linkage was obtained ( $F=10.17, \quad$ df $=1 / 120, \quad p<.005)$, indicating that more extreme ratings of the stimulus person were obtained when the information about the stimulus person was conveyed via an associative link, as opposed to a dissociative link. Since the positive trait words utilized in the experiment were just as positive as the negative trait words were negative, no main effect for information was hypothesized and, in effect, no main effect for information was obtained $(\mathrm{F}<1)$. terms of the weight parameter. That is, information conveyed via dissociative links may have reduced weight or importance in the overall judgments, and hence, in the case of the present experiment, the evaluation of the stimulus object when dissociative links are used to convey information would not be as extreme.

Although the results support the

The results support the hypothesis that if one wishes to produce extreme positive ratings of a stimulus person, it is better to link the stimulus person associatively to a positive trait word (e.g., Mr. X is reasonable) than it is to link him dissociatively to the corresponding negative trait word (e.g., Mr. X is not unreasonable). Alternatively, if one wishes to produce extreme negative ratings of a stimulus person, it is better to link him associatively to a negative trait word (e.g., Mr. $\mathrm{X}$ is unreasonable) than it is to link him dissociatively to the corresponding positive trait word (e.g., Mr. X is not reasonable).

Perhaps these results can best be interpreted from an informational point of view. Stating that Mr. $X$ is reasonable or that Mr. X is unreasonable gives the $S$ more definitive information about the stimulus object than stating that Mr. X is not unreasonable or that $\mathrm{Mr} . \mathrm{X}$ is not reasonable. In the situation where Mr. $\mathrm{X}$ is described as not unreasonable, all that the Ss know is that $\mathrm{Mr}$. $\mathrm{X}$ is not unreasonable. This does not definitively imply that he is reasonable, and hence the $S s$ have less information about the stimulus object in this situation. Amount of information, according to Fishbein (1963), is related to intensity of affective response.

The results can also be interpreted in terms of Anderson's (1968b) model for information integration. According to Anderson's model, each stimulus item can be represented by two parameters. One of these would be the value of the stimulus item along some dimension of judgment (e.g., like-dislike) and the other would be the importance (weight) of the stimulus in the overall judgment. The linkage variable in the present experiment could be interpreted in position that more extreme ratings of a stimulus person may be obtained by utilizing associative links to convey information about the stimulus person, one must be cautious in generalizing these results.

The information about the stimulus person was presented in the format of $\mathrm{Mr} . \mathrm{X}$ is (is not) trait word (positive or negative). The stimulus person in this study is not presenting information about himself but rather is having information conveyed about him via a second party-in this case, the $E$. It may be that in situations where the stimulus person conveys the information about himself (e.g., Mr. X has stated "I am reasonable" vs "I am not unreasonable"), associative links may work to the disadvantage of the stimulus person-in this case, indicating a "bragging effect." Research on this question is currently in progress.

\section{REFERENCES}

ABELSON, R. P. \& ROSENBERG, M. J Symbolic psychologic: A model of attitudinal cognition. Behavioral Science, $1958,3,1-13$.

ANDERSON, N. H. Likeableness ratings of 555 personality-trait-words. Journal of Personality \& Social Psychology, $1968 \mathrm{a}$. 9, 272-279

ANDERSON, N. H. A simple model for information integration. In R. P. Abelson. E. Aronson, W. J. McQuire, T. M. Newcomb, $M$, Rosenberg, and $P$. Tannenbaum (Eds.), Theories of cognitive consistency: A sourcebook. Chicago: Rand McNally, $1968 \mathrm{~b}$.

FISHBEIN, M. An investigation of the relationships between beliefs about an object and the attitude toward that object. Human Relations, 1963, 16. 233-239.

HEIDER, F. The psychology of interpersonal relations. New York: Wiley, 1958.

OSGOOD, C. E., \& TANNENBAUM, P. H. The principle of congruity in the prediction of attitude change. Psychological Review, 1955, 62, 42-55. 